and health characteristics. Health and well-being outcomes included: self-rated health measured on a five-point scale, and life satisfaction, feeling that life is worthwhile, happiness, and anxiety, measured on eleven-point Likert scales. We conducted a causal mediation analysis to quantify natural indirect effects representing how much of the effect of disability acquisition on each outcome was explained by barriers to participation including employment, economic life, transport, leisure activities, social contact and accessibility. We used multiple imputation with 50 imputed datasets to account for missing data and conducted analyses in Stata/SE 15.

Results There was evidence that people who had recently acquired a disability had poorer health and well-being compared to people with no disability. Barriers to participation explained 13\% (95\% CI 11\%, 14\%) of inequalities in selfrated health, and were higher for all measures of well-being: life satisfaction $(43 \%, 95 \%$ CI 39\%, 47\%), feeling that life is worthwhile $(36 \%, 95 \%$ CI 31\%, 40\%), happiness (46\%, 95\% CI 39\%, 53\%) and anxiety (27\%, 95\% CI 24\%, 31\%).

Conclusion Despite methodological limitations including strong assumptions about confounding and potential selection bias from missing data, this is the first study to quantify how much of the inequalities in health and well-being between people with and without disabilities are explained by social barriers to participation. We found that a substantial proportion of the inequalities in health and well-being experienced by people with recently acquired disabilities were driven by social barriers to participation. The findings that some of these differences are socially produced have important policy implications, highlighting modifiable factors amenable to public health interventions to target the mechanisms causing the health inequalities.

\section{P63 ADVERSE PREGNANCY OUTCOMES AND LONG-TERM RISK OF MATERNAL RENAL DISEASE: A SYSTEMATIC REVIEW AND META-ANALYSIS}

\begin{abstract}
${ }^{1,2}{ }^{2} \mathrm{M}$ Barrett* ${ }^{*}{ }^{2} \mathrm{FP}$ McCarthy, ${ }^{3} \mathrm{~K}$ Kublickiene, ${ }^{4} \mathrm{~S}$ Cormican, ${ }^{4} \mathrm{C}$ Judge, ${ }^{3} \mathrm{M}$ Evans, ${ }^{5} \mathrm{M}$ Kublickas, ${ }^{1}$ IJ Perry, ${ }^{3} \mathrm{P}$ Stenvinkel, ${ }^{1,2} \mathrm{AS}$ Khashan. ${ }^{1}$ School of Public Health, University College Cork, Cork, Ireland; ${ }^{2}$ Irish Centre for Fetal and Neonatal Translational Research (INFANT), University College Cork, Cork, Ireland; ${ }^{3}$ Department of Clinical Science, Intervention and Technology (CLINTEC), Karolinska Institutet, Stockholm, Sweden; ${ }^{4}$ Department of Nephrology, University Hospital Galway, Galway, Ireland; ${ }^{5}$ Department of Obstetrics and Gynaecology, Karolinksa Institutet, Stockholm, Sweden
\end{abstract}

\subsection{6/jech-2019-SSMabstracts.214}

Background Adverse pregnancy outcomes, like hypertensive disorders of pregnancy (HDP), gestational diabetes (GDM) and preterm delivery, are associated with increased risk of long-term maternal cardiovascular and cerebrovascular disease. Comparatively little is known about whether adverse pregnancy outcomes increase the risk of maternal renal disease. We aimed to investigate associations between adverse pregnancy outcomes (HDP, GDM, preterm delivery) and long-term maternal chronic kidney disease (CKD) and end-stage kidney disease (ESKD), by synthesising the results of relevant published studies.

Methods A systematic search of PubMed, EMBASE and Web of Science was undertaken from inception of databases to 31 July 2018. Case-control and cohort studies published in English were eligible for inclusion if they provided original effect estimates for associations between adverse pregnancy outcomes (HDP, GDM, preterm delivery) and maternal renal disease (primary outcomes: CKD, ESKD; secondary outcomes: renal hospitalisation, mortality due to renal disease). Two independent reviewers extracted data and assessed risk of bias. Random effects meta-analyses were conducted using RevMan 5.3 to determine the pooled adjusted odds ratio (AOR) and $95 \%$ confidence interval $(95 \% \mathrm{CI})$ for each association between each adverse pregnancy outcome and CKD or ESKD respectively. Subgroup analysis by HDP subtype was performed.

Results Of 5,120 studies retrieved, 21 studies met inclusion criteria (37 adjusted effect estimates in total, including 4,483,847 participants). HDP was associated with significantly increased odds of ESKD (AOR 6.58, 95\%CI 4.06-10.65, based on nine effect estimates), CKD (AOR 2.08, 95\%CI 1.06-4.10, based on eight estimates), and renal hospitalisation (AOR 2.29, 95\%CI 1.42-3.71, based on six estimates). The magnitude of association was dependent on HDP subtype: AOR for preeclampsia and ESKD was 4.87 (95\%CI 3.017.87); for gestational hypertension and ESKD was $3.65(95 \%$ CI 2.34-5.67); for other HDP (including chronic hypertension \pm superimposed preeclampsia) and ESKD was $14.67(95 \% \mathrm{CI}$ 3.21-66.97). Preterm delivery was associated with increased odds of ESKD (AOR 2.16, 95\%CI 1.64-2.85, based on three estimates). GDM was associated with increased odds of CKD among black women (AOR 1.78, 95\%CI 1.18-2.70), but not white/Caucasian women (AOR 0.81, 95\%CI 0.58-1.13, based on four estimates).

Conclusion Women who experience adverse pregnancy outcomes have increased odds of long-term renal disease, particularly those exposed to HDP. This study was limited by small numbers of studies in each individual meta-analysis, restricting the ability to assess for publication bias. Long-term follow-up should be optimised for women who experience adverse pregnancy outcomes, and preventive interventions may be warranted to reduce their risk of clinically significant renal disease.

\section{P64 SELF-HARM AMONG THE HOMELESS POPULATION IN IRELAND: A NATIONAL REGISTRY-BASED STUDY OF INCIDENCE AND ASSOCIATED FACTORS}

${ }^{1} \mathrm{PM}$ Barrett ${ }^{*},{ }^{2} \mathrm{E}$ Griffin, ${ }^{2} \mathrm{P}$ Corcoran, ${ }^{1} \mathrm{MT} \mathrm{O}$ 'Mahony, ${ }^{2} \mathrm{E}$ Arensman. ${ }^{1}$ Department of Public Health, St. Finbarr's Hospital, Cork, Ireland; ${ }^{2}$ National Suicide Research Foundation, University College Cork, Cork, Ireland

\subsection{6/jech-2019-SSMabstracts.215}

Background Suicide rates are higher among the homeless population than the general population, and the homeless are recognised as a priority group for suicide prevention. Self-harm is a strong predictor of future suicide, particularly repetition of self-harm. Little is known about the incidence of self-harm, and its associated predictive factors, among the homeless. The purpose of this study was to quantify the burden of self-harm among the homeless population in Ireland, and to assess factors associated with self-harm and repeated self-harm.

Methods Data on self-harm presentations to all 34 hospital emergency departments in Ireland were collected by the National Self-Harm Registry Ireland (NSHRI). Index and repeat presentations from 2010-2014 were included for the homeless and fixed residence populations. Individuals with no fixed abode, or who lived in recognised accommodation for the homeless, were recorded as being homeless. Age-standardised incidence rates (ASIR) of self-harm were calculated using NSHRI data and national population estimates from the 